\title{
Analysis on Accurate Early Warning and Intervention Model of Blended Learning in Ideological and Political Courses Based on Learner Portrait
}

\author{
Yang Zhang, ${ }^{1, *}$ \\ ${ }^{1}$ School of Marxism, Hunan Agricultural University, Changsha, Hunan 410128, China \\ *Corresponding author. Email: zhangyang66@hunau.edu.cn,37735349@qq.com
}

\begin{abstract}
To improve the teaching effect of ideological and political courses, learning early warning and intervention is the key to it. On the basis of learning analysis, this paper puts forward the process and principle of constructing learner portrait, and design the "BILDES" six-side learner portrait model, which includes six dimensions: basic information, internal characteristics, learning style, learning behavior, emotional attitude and social interaction. Based on the portraits of learners, an accurate early-warning and intervention model of blended learning of ideological and political courses is constructed from the theoretical level. In the earlywarning mode, warnings are given to students through red lights or green lights, emails, digital dashboards and news push. In the intervention mode, systematic automatic intervention and manual intervention are combined. Through accurate identification, effective early warning, accurate and individualized intervention, the learning crisis can be effectively resolved and the quality of ideological and political courses can be improved.
\end{abstract}

Keywords: ideological and political theory course; blended learning; learner portrait; learning warning;

learning intervention

\section{INTRODUCTION}

With the rapid advancement of big data, cloud computing and artificial intelligence technology and the widespread rise of MOOCs, "Internet +"education has entered the educational horizon with its unique charm, bringing about great changes in teaching and learning. In particular, during the period of COVID-19 prevention and control in 2020, the revolutionary development of online teaching, Chinese universities offered students 1.1 million courses, a total of 17.19 million courses; There were 22.59 million college students participated in online learning, with a total of 3.5 billion person-times [1]. Massive learning data burst and gather, which contains significant teaching value. The blended learning mode, which combines the advantages of traditional classroom teaching methods with the advantages of online learning, has been widely used in ideological and political teaching in colleges and universities. Mining the great teaching value of the integration and innovation of big data, artificial intelligence technology and ideological and political education and giving full play to the advantages of blended teaching have become the future development trend of ideological and political teaching. Ideological and political theory course is the key to carry out the fundamental task of moral education, and is the main channel and the central site of ideological and political education for college students. The <Some opinions on deepening the reform and innovation of school ideological and political theory course in the new era >which was issued by the General Office of the Communist Party of China and the State Council General Office of China explicitly put forward:" To vigorously promote the reform of education courses teaching method, promote education courses teachers' information ability quality, promote the artificial intelligence and other modern information technology in education courses teaching applications." [2] Learning early warning and intervention is the key to improve the teaching effectiveness of ideological and political courses, change "lood irrigation" to" precise drip irrigation", master learners' learning conditions. In combination with the new situation, new requirements and new characteristics of ideological and political course teaching in colleges and universities, actively explore the application of information technology combined with traditional ways of accurate early warning and intervention of blended learning in ideological and political course, which is of great significance to enhance the attractiveness of ideological and political course, promote independent and personalized learning of learners, and improve the effectiveness of ideological and political education. 


\section{THE CONSTRUCTION OF THE LEARNER PORTRAIT OF THE BLENDED LEARNING IN IDEOLOGICAL AND POLITICAL COURSES}

The value of educational big data is to solve the "cognition" problem of learners and to complete the accurate learner portrait. With the vigorous development of online teaching and blended teaching of ideological and political courses, data accumulation is adequate and technology is constantly mature. The whole process of individual learning behavior can be objectively quantified through the "datamation" of its information footprint, which provides a data basis for tracking, modeling, warning and intervention. The essence of the learner portrait of the blended learning in ideological and political courses is still the description, analysis and presentation of the characteristics of learners. With the help of the application potential of user portrait and based on learning analysis, the big data of education is labeled and visualized, and the characteristics of learners are analyzed in a multidimensional way to have a deep understanding of learners [3]. Grasp the learner's personality learning preferences, combined with offline classroom teachers' direct observation and face-to-face communication, experience judgment, to identify high-risk learners and lay the foundation for more accurate learning warning and intervention.

\subsection{Dimension analysis on the learner portrait of the blended learning in ideological and political courses}

In combination with the blended teaching characteristics of ideological and political courses, the learner portrait should be carried out around the following six dimensions, and constructed the "BILDES" six-side portrait model:

(1)Basic Information Dimension: name, gender, age, learning experience...;

(2)Internal Characteristics Dimension: personality, personality bias, purpose, cognitive characteristics... ;

(3)Learning Style Dimension: learning resource types and content preferences, preliminary knowledge base (beginners/contact/good foundation), learner type (stepby-step/interested/problem solving/testoriented/diverging distraction/Learning difficulties, etc.), learning methods...;

(4)Dimension of Learning Behavior: schedule, online time, learning time, information habits, facial expression recording, learning process... ;

(5)Emotional Attitude Dimension: psychological identity, learning emotion (positive emotion/negative emotion), learning Attitude (positive Attitude/negative Attitude), learning confidence, learning motivation, learning interest, learning will... ;
(6)Social Interaction Dimension: discussion and participation, posting and sharing, exchange and comment, peer interaction, teacher-student interaction... .

\subsection{Principles and process of constructing learner portrait of blended learning in ideological and political courses}

\subsubsection{Principles of constructing learner portrait of blended learning in ideological and political courses}

\subsubsection{Value, objectivity and legitimacy of data sources}

The learner portrait of blended learning in ideological and political courses emphasis on "Educational contextuality" and "Value Density", also need to combine online data collection and offline classroom communication observation. Through three main methods of social investigation, network data collection and perception technology collection, multi-modal data of the whole process of learners can be obtained to ensure the value, objectivity and authenticity of portrait data. Social survey is represented by the methods of interview, observation and questionnaire survey, which supports capturing the representation data of learners psychological characteristics, advanced abilities and literacy. Online data collection supports capturing online learning data that trace learners' learning behavior, data mining and semantic analysis. [4] With the help of intelligent terminals or wearable devices, perceptual technology can grasp the interactive data, such as picture, text, audio image, visual sense of touch, etc., to track and analyze learners' learning behavior, emotion, interest and attention, such as analyzing learners' brain wave patterns by means of the electroencephalogram experiment, to obtain learners' attention and interest profile. [5] At the same time, data privacy protection should be paid attention to during data collection to ensure the legitimacy of data collection and application.

\subsubsection{Method fusion and path fusion in modeling process}

Different from the previous learning analysis methods, the modeling process of learner portrait integrates quantitative and qualitative methods, focusing on quantitative analysis and paying attention to data support and mutual verification between different data. The quantitative method is to conduct detailed statistical analysis and 
calculation of learner data, so as to accurately explore learning needs. The qualitative method can analyze the educational scene and the learner's mind, so as to abstract and summarize the nature and characteristics of the user, which can be used to give semantic meaning to the generated labels. The key to the learner portrait is to add "tags" to the student, each of which is a highly refined feature identifier, and finally to combine all the tags to create a three-dimensional portrait of the student.

At the same time, due to the education courses hybrid teaching is organic combination of online and offline, and as a result, when modeling in addition to use online education platform to collect data for portraits, based on the offline in the classroom teachers and students to observe, face to face communication, experience, etc., can also be validated portraits of learners, supplement and correction, accurate and perfect.

\subsubsection{Hierarchy and visualization of modeling output}

Due to the attention of the learner's individual and social reality in education, the modeling output of learner portrait should also show the hierarchy of individual portrait and group portrait. It is necessary not only to draw the individual portrait of students, but also to draw the whole portrait of the students in a particular class, and to make a cluster analysis of the students in the same class to form the portrait of different groups of students. Then the visual output can accurately identify and distinguish the common needs and differentiated needs of different students, and lay an objective foundation for the implementation of precision teaching. The process of constructing the learner portrait of the blended learning in ideological and political courses. Based on the online education platform suitable for ideological and political course teaching, the learning behavior data collection and processing are completed. The construction process of learner portrait is designed, which is divided into four steps: (1) Data collection; (2) Data analysis; (3) Learner feature extraction to form "labels"; (4) Construction and output of learner portrait model.

\section{CONSTRUCTION OF EARLY WARNING AND INTERVENTION MODEL OF BLENDED LEARNING IN IDEOLOGICAL AND POLITICAL COURSES BASED ON LEARNER PORTRAIT}

In the context of modern education concepts, it gives full play to the advantages of blended learning in ideological and political courses, makes use of online education platform, and utilizes multi-modal methods such as learning analysis technology, artificial intelligence and data mining to collect and screen, mine and analyze the learning behavior data of the whole learning process and learning results. Based on the learner portrait, multi-level, multidirectional, diversified and personalized early-warning and intervention strategies and measures are adopted to construct a precise early-warning and intervention model for blended learning of ideological and political courses (Figure 1).

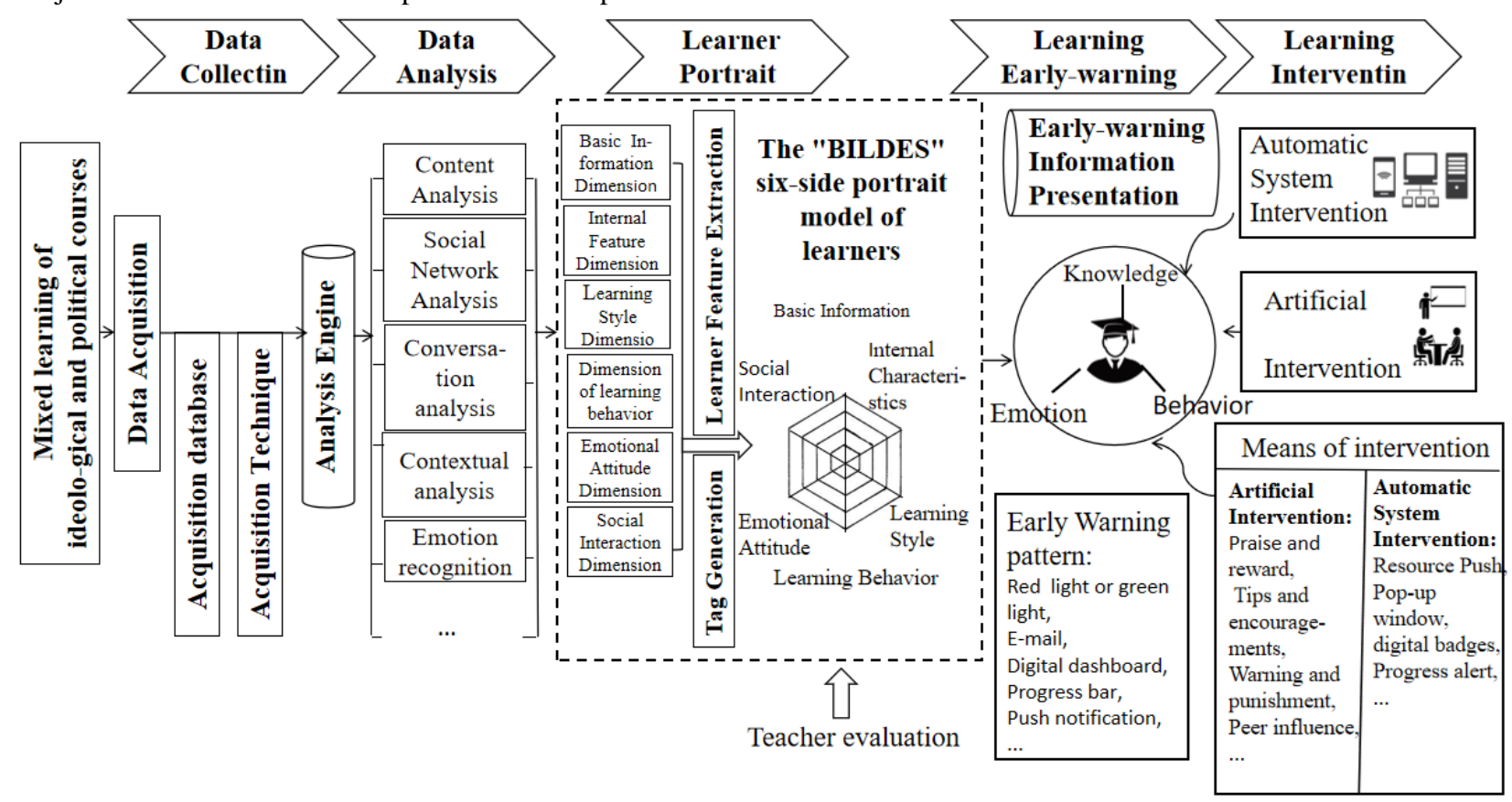

Figure 1. The early warning and intervention model of blended learning in ideological and political courses based on learner portrait 
Based on the supply of high-quality resources, the model uses data mining and analysis tools to collect a large number of students' learning behavior data from online education platform and information-based teaching tools. Multi-modal analysis such as content analysis, social network analysis, conversation analysis, context analysis and emotion recognition was carried out based on data collection. Combined with the teachers' subjective evaluation of classroom teaching, the "BILDES" Six-sided Learner Portrait is made from six dimensions: basic information, internal characteristics, learning style, learning behavior, emotional attitude and social interaction. The analysis results are output in the form of charts, figures and so on by visualization technology. According to the learning situation presented by the analysis results and the students' online and offline learning performance, crisis diagnosis is made to judge whether there is a learning crisis in the current stage of learners. Different teaching strategies are adopted for different groups and individuals of students. At the same time, the diagnosis of students' learning crisis is carried out in a cycle with the continuous development of learning activities. With the continuous updating of the collected data, students' learning situation can be analyzed in real time, their learning progress can be monitored at all times, and hierarchical teaching, differentiated teaching and personalized teaching can be carried out according to students' learning situation.

When students may have a learning crisis, appropriate early warning and intervention measures should be provided in time to help students successfully complete the course learning and ensure the quality of the course learning. In the early-warning mode, warnings are given to students through red lights or green lights, emails, digital dashboards, and news push, so that students can understand the completion of their learning activities and accurately evaluate their learning results. In the intervention mode, systematic automatic intervention is combined with manual intervention. In the manual intervention, teachers praise and reward, prompt and encourage, warning and punishment, combined with peer influence, to encourage students to speed up the learning progress and actively complete the learning task. Automatic intervention of the system is mainly carried out through resource push, pop-up window, digital badge, progress reminder, etc. [6]. Push to students includes the choice of learning partners, the supplement of learning resources and the use of learning tools, which can be used to optimize the learning process and improve learning efficiency. The pop-up window is used to provide the students with the test questions of the related learning content, to urge the students to carry on the self-determination test, to discover the learning blind spot and to make up in time, so as to ensure the students' learning amount. In the form of digital badges to recognize the efforts of students, stimulate students' learning interest, enhance students' learning motivation, promote active learning; progress reminder to achieve self-monitoring, enhance learning sustainability [6]. Self-monitoring of progress reminders to enhance sustainability of learning.

\section{EFFECT ANALYSIS OF ACCURATE EARLY WARNING AND INTERVENTION MODEL OF BLENDED LEARNING IN IDEOLOGICAL AND POLITICAL COURSES BASED ON LEARNER PORTRAIT}

In order to test the feasibility and validity of the accurate early warning and intervention model of blended learning in ideological and political course, we take "the course of the ideological and moral cultivation and legal basis" as the experimental object. Taking the data of student archives, online learning platform and classroom learning behavior as data sources, the sample data were divided into training samples and test samples for actual analysis and portrait of learners. Through the data collection and analysis of the learning platform system, 6 medium-risk learners and 4 high-risk learners were identified. Based on the above analysis results, combined with classroom observation and students' actual learning effectiveness, we found through early warning that the identified risk learners did have learning risks in the course learning performance, which indicates that the early warning model and system analysis based on learner portrait have high validity. Through this early warning model and system, we can quickly identify students who are at risk in the performance of course learning outcomes, and we can grasp the performance, characteristics and causes of inefficient learning in the learning process through learner portraits, to find out the weak points in the learning process, and to provide reference basis for the later stage teachers to carry out precise teaching intervention. By dividing medium-risk learners and high-risk learners into 2 groups, each group has 3 medium-risk learners and 2 high-risk learners. The experimental group received precise learning intervention, while the control group did not. After a 4-week experiment, all the medium-risk learners in the experimental group became low-risk learners, one of the two high-risk learners became low-risk learners, and one became medium-risk learners through systematic automatic intervention and teacher communication intervention. In the control group, the learning activity did not increase but decreased over time without learning intervention. The risk level of 2 highrisk learners was increased, the learning risk of 2 mediumrisk learners remained unchanged, and 1 even became a high-risk learner. Preliminary experiments show that the model can promote accurate identification, effective early warning and accurate intervention to a certain extent, which is helpful to resolve learning crisis and improve learning quality. In addition, combining the technical characteristics of real-time updating of educational big data monitoring and updating the students' data in time and adjusting the early warning state dynamically can form the normal and periodic monitoring of learning performance. Although the accurate early warning and intervention model of blended learning in ideological and political education based on Learner's portrait can identify learners with learning risk to a certain extent, and is beneficial to 
the development of accurate and effective intervention, however, only a small sample of the initial experiments, the follow-up needs to be larger samples, refinement, more rounds of empirical research to further verify and amend.

\section{CONCLUSION}

Under the situation of education modernization and the normalization of epidemic prevention and control, the blended learning in ideological and political courses has a broad application prospect, and the importance of learning accurate early warning and intervention is becoming increasingly prominent. The diversity of learning fields, situations and ways requires the integration and analysis of data in different learning environments. The uniqueness of ideological and political courses requires that more attention should be paid to the role of students' emotions in learning in the future study analysis and research, so as to promote the real unity of students' knowledge, emotion, intention and behavior, and promote the realization of wisdom education, personality learning and lifelong learning. Relying on online education platform, by the early warning and intervention model, we can based on education courses blended learning behavior data generation learners portrait, quickly identify exist different degree of risk in the course aspects of student individual or group, and find out its characteristics and weak spot in the learning process, through the system and the artificial diversity, multi-dimensional, accurate early warning and intervention, Effectively resolve the learning crisis, for the implementation of precision teaching, personalized teaching, improve the effectiveness of the reference. In this study, a precise early-warning and intervention model of blended learning in ideological and political courses was constructed from the theoretical level. On this basis, several rounds of empirical studies were conducted to iteratively revise the model. At the same time, the scientific, accurate and effective learning early warning and intervention also requires the cooperation of the government, schools, teachers, students and technical parties. In the future, the application scenarios and strategies of the model will be discussed from different levels and perspectives.

\section{ACKNOWLEDGMENT}

The work described in this paper was fully supported by a grant from the science research project of Education Department of Hunan Province "A study on the precise early-warning and intervention mechanism of blended learning based on the perspective of learning analysis" (No 18C0136), and the research project of ideological and political construction of ordinary higher education in Hunan Province" Research and Practice on the Coconstruction and Sharing mechanism of Ideological and political Teaching resources of University Courses based on“" Internet +"(No. HNKCSZ-2020-0187).

\section{REFERENCES}

[1]Ministry of Education: Turning "wartime" online education "novelty" into "new normal", 2020. DOI:http://www.chinanews.com/gn/2020/0827/9275395.shtml (In Chinese).

[2]Xinhuanet. The General Office of the Communist Party of China (CPC) Central Committee and The General Office of the State Council issued <Several Opinions on Deepening the Reform and Innovation of Ideological and Political Theory Courses in Schools in the New Era>, 2019. DOI:

http://www.xinhuanet.com/politics/2019-

08/14/c_1124876294.htm(In Chinese).

[3]Xiao Jun, QIAO Hui, Li Xuejiao. The construction of online learner portrait in big data environment, Open Education Research, vol. 25, pp111-120, August 2019. DOI:DOI:10.13966/j.cnki.kfjyyj.2019.04.012(In Chinese).

[4]Mo Wei. Study on the construction and application of learner's portrait, Journal of Hunan Institute of Science and Technology (Natural Sciences), vol. 34, pp 6469,September 2021. DOI:10.16740/j.cnki.cn431421/n.2021.03.013(In Chinese).

[5]CHEN Haijian, DAI Yonghui, HAN Dongmei, FENG Yanjie \& HUANG Hexiao. Learner's Portrait and Individualized Teaching in Open Education, Open Education Research,Vol.23,pp105-112,June 2017,DOI:10.13966/j.cnki.kfjyyj.2017.03.012(In Chinese).

[6]ZHAO Huiqiong, JIANG Qiang, ZHAO Wei, LI Yongfan, ZHAO Yan. Empirical Research of Predictive Factors and Intervention Countermeasures of Online Learning Performance on Big Data-based Learning Analytics, E-education Research, vol. 38, pp 62-69, January 2017. DOI:10.13811/j.cnki.eer.2017.01.008 (In Chinese). 